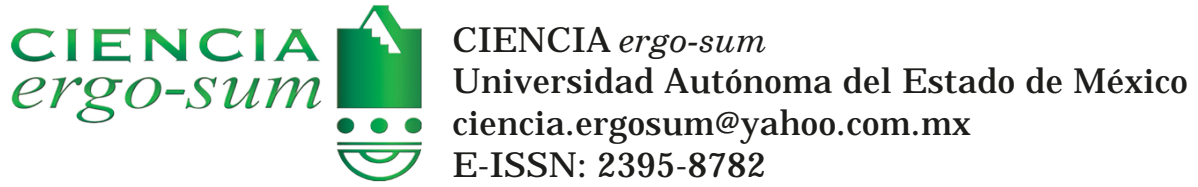

\title{
Aprovechamiento de cáscaras de frutas: análisis nutricional y compuestos bioactivos
}

Vargas y Vargas, María de Lourdes; Figueroa Brito, Héctor; Tamayo Cortez, J orge Abraham; Tol edo López, Víctor Manuel; Moo Huchin, Víctor Manuel Aprovechamiento de cáscaras de frutas: análisis nutricional y compuestos bioactivos

CIENCI A ergo-sum, vol. 26, núm. 2, julio-octubre 2019| e52

Universidad Autónoma del Estado de México, México

Esta obra está bajo una Licencia Creative Commons Atribución-NoComercial-SinDerivar 4.0 Internacional.

Vargas y Vargas, M. de L., Figueroa Brito, H., Tamayo Cortez, J. A., Toledo López, V. M. y Moo Huchin, V. M. (2019). A provechamiento de cáscaras de frutas: análisis nutricional y compuestos bioactivos. CIENCIA ergo-sum, 26(2). https://doi.org/10.30878/ces.v26n2a6 


\title{
Aprovechamiento de cáscaras de frutas: análisis nutricional y compuestos bioactivos
}

Using of fruits peel: nutritional analysis and bioactive compounds

\author{
Maria de Lourdes Vargas y Vargas \\ Tecnológico Nacional de México/IT Mérida, México \\ acras_99@yahoo.com \\ Héctor Figueroa Brito \\ Tecnológico Nacional de México/IT Mérida, México \\ hectorfigueroa_13@hotmail.com \\ Jorge Abraham Tamayo Cortez \\ Tecnológico Nacional de México/IT Mérida, México \\ jtamayin@hotmail.com \\ Victor Manuel Toledo López \\ Tecnológico Nacional de México/IT Mérida, México \\ vtoledo08@yahoo.com.mx \\ Victor Manuel Moo Huchin \\ Tecnológico Nacional de México/IT Mérida, México \\ vmmoo@yahoo.com
}

Recepción: 18 de diciembre de 2017

Aprobación: 25 de abril de 2018

\section{Resumen}

Se analiza el contenido bromatológico y bioactivo de residuos agroindustriales provenientes de frutas y vegetales con el fin de darle un valor agregado. Se utilizaron cáscaras de papaya, zanahoria, berenjena y lima, las cuales fueron secadas previamente, molidas y tamizadas para luego determinar la humedad, cenizas, fibra, proteína, extracto etéreo, así como la extracción y cuantificación de compuestos tales como carotenos, polifenoles totales, flavonoides, antocianinas y la capacidad antioxidante. Los resultados indicaron que las cáscaras de papaya y de berenjena presentaron las mejores características de calidad bromatológicas para cenizas (10.45 y 9.2\%), proteína (2.23 y $13.50 \%)$, fibra $(0.643$ y $1.88 \%$ ) y capacidad antioxidante $(99.63$ y $97.90 \%)$ con las que se podrían desarrollar productos de utilidad en la industria alimentaria o farmacéutica.

PALABras ClaVes: cáscaras, análisis bromatológicos, antioxidantes, compuestos bioactivos.

\section{Abstract}

The main objective of this work was to analyze the bromatological and bioactive content of agroindustrial waste from fruits and vegetables in order to give it an added value. For that, papaya, carrot, eggplant and lime peel were used, which were previously dried, ground and sieved in order to determine the humidity, ash, fiber, protein, ether extract, as well as the extraction and quantification of compounds such as carotenes, total polyphenols, flavonoids, anthocyanins and antioxidant capacity. The results indicated that the papaya and eggplant peel presented the best bromatological quality characteristics for ash (10.45 and $9.2 \%$ respectively), protein (2.23 and $13.50 \%$ ) and fiber (0.643 and $1.88 \%$ ) and antioxidant capacity ( 99.63 and $97.90 \%$ respectively) with which useful products could be developed in the food or pharmaceutical industry.

KEYWORDS: peel, bromatological analysis, antioxidants, bioactive compounds.

\section{INTRODUCCIÓN}

En la actualidad el desarrollo de la industria conlleva a la producción de residuos y, por consiguiente, a implementar y perfeccionar nuevas técnicas o métodos para un mejor aprovechamiento. En el proceso de los alimentos, además del producto deseado, se generan subproductos, residuos y productos fuera de norma (Méndez, 1995), cada uno de 
los cuales puede servir para consumo humano o animal y aplicación industrial, lo que traería beneficios económicos. Debido a las exigencias crecientes del mercado, la transformación de los productos agrícolas se ha convertido en parte importante de los procesos industriales dentro de muchas empresas. Dichos procesos ocasionan de forma ineludible residuos causantes de contaminación ambiental en aguas, suelos y atmósfera, que además ponen en peligro la salud humana y el nicho ecológico de muchas especies animales y vegetales. Conscientes de esta problemática, varios investigadores han dedicado sus esfuerzos a evaluar las propiedades y usos de estos materiales con el fin de mitigar su impacto ambiental, además de buscar posibles aplicaciones industriales que representen beneficios económicos. Algunas de las áreas dentro de las que se han centrado estas investigaciones es en la obtención de combustibles (Parawira, 2008), de saborizantes y compuestos aplicables a la industria de alimentos (Rivas et al., 2009; DiGioia et al., 2007) y de pesticidas (Cayuela et al., 2008), entre otras. Asimismo, la utilización de residuos agroindustriales, como materia prima de bajo costo para obtener productos químicos finos, representa una opción para transformar los desechos en nuevas materias primas que se perfilan como una alternativa atractiva para conseguir compuestos con propiedades benéficas. Sin embargo, los residuos generados por las transformaciones agroindustriales y por las pérdidas poscosecha en nuestro país aún no han sido aprovechados eficientemente, en parte, porque su valor es desconocido y sobre todo por la falta de métodos apropiados para la preparación y caracterización de sustancias de mayor valor agregado, las cuales contengan la suficiente calidad e inocuidad como para ser usadas en esos procesos. Por este motivo, los procesos oxidativos han sido relacionados con diversas afecciones, como las que involucran deterioro del sistema nervioso central (Angulo et al., 2001), y además son causantes de alteraciones de múltiples productos dentro de la industria alimenticia debido al detrimento de sus propiedades organolépticas. Como respuesta, este sector productivo debe recurrir al uso de algunos agentes antioxidantes de origen sintético como el hidroxitolueno butilado y el hidroxianisol butilado, responsables de daños en el hígado y de la carcinogénesis (Wichi, 1988). El papel que tienen los vegetales y productos alimenticios en la prevención de enfermedades ha sido atribuido a las propiedades antioxidantes de sus compuestos polifenólicos. Con este conocimiento, se trata de demostrar el valioso potencial de algunos materiales subutilizados o de escaso interés comercial como fuente de compuestos antioxidantes y antimicrobianos (Oliveira et al., 2009). Estos residuos representan una alternativa para la formulación de alimentos funcionales libres de productos químicos sintéticos al aprovechar su capacidad antioxidante con lo que se lograría disminuir el porcentaje de desechos provenientes de la industria.

Por lo anterior, el objetivo de este artículo es elaborar un análisis nutrimental y antioxidante de algunos desechos de cáscaras de frutas con el fin de darle un valor agregado y posteriormente pueda aplicarse en el desarrollo de productos alimenticios o farmacéuticos.

\section{Materiales y métodos}

\section{1. Materia prima}

Las frutas seleccionadas para el estudio fueron papaya (Carica papaya), zanahoria (Daucus carota sub. Sativus), berenjena (Solanum melongena) y lima (Citrus aurantifolia) en estado de madurez para consumo. Después de separar la pulpa, la cáscara se puso a secar en una estufa por 24 horas a una temperatura de $45^{\circ} \mathrm{C}$, la cual fue elegida con el fin de evitar la degradación de los compuestos bioactivos. Una vez realizado este paso, se trituró en una licuadora hasta obtener un polvo fino.

\section{Análisis Químicos}

Los análisis bromatológicos que se llevaron a cabo fueron el de humedad, cenizas, proteína total grasa y fibra, en tanto que los compuestos bioactivos cuantificados fueron los fenoles, $\beta$-carotenos, antocianinas, flavonoides y capacidad antioxidante. Las muestras en cada análisis se trabajaron por triplicado. 


\section{1. Humedad}

El porcentaje de humedad se determinó mediante la metodología del AOAC (934.06). Se pesaron $2 \mathrm{~g}$ de muestra y se colocaron en crisoles previamente acondicionados a peso constante, los cuales se colocaron en una estufa durante 12 horas a una temperatura de $90{ }^{\circ} \mathrm{C}$. El porcentaje de humedad se determinó por diferencia de peso.

\subsection{Cenizas}

Para determinar las cenizas se siguió el método 923.03 de la AOAC. Se calcina/incinera la muestra tras su desecación a $550^{\circ} \mathrm{C}$ en el horno mufla y se calcula el residuo de incineración por diferencia de peso. Los resultados se expresan como porcentaje de cenizas.

\subsection{Proteína total}

El contenido de proteína se evaluó por medio del método de Kjeldahl (AOAC 920.53), el cual se basa en la digestión de las proteínas y otros componentes orgánicos del polvo de las cáscaras en una mezcla con ácido sulfúrico en presencia de catalizadores. El resultado del análisis representa el contenido de proteína cruda del alimento, ya que el nitrógeno también proviene de componentes no proteicos. Para expresar el contenido en proteínas es necesario multiplicar la cantidad de nitrógeno por un factor variable según el tipo de muestra analizada, que para frutas y verduras es de 6.25 .

\subsection{Grasa}

El contenido de extracto etéreo se determinó de acuerdo con la AOAC mediante la extracción Soxhlet utilizando éter de petróleo como solvente. Se efectuó la extracción durante 4 horas. El resultado se expresa como porcentaje de extracto etéreo.

\subsection{Fibra}

El método empleado para establecer la fibra alimentaria total, soluble e insoluble fue el de la AOAC (991.43), que permite determinar las sustancias orgánicas libres de grasas e insolubles en medio ácidos y alcalinos, denominadas convencionalmente fibra bruta. El resultado se expresó como porcentaje de fibra.

\subsection{Extracto libre de nitrógeno}

Se obtiene cuantitativamente con el remanente de restarle a $100 \%$ de la muestra. La suma obtenida en los análisis de humedad, cenizas, proteína, extracto etéreo y fibra bruta. El resultado se expresa como porcentaje de ELN.

\subsection{Determinación de polifenoles totales}

Se llevó a cabo por el método descrito por Folin y Ciocalteu (Prior et al., 2005; Mullen et al., 2007). La absorbancia se leyó a $765 \mathrm{~nm}$. Los fenoles totales se calcularon como equivalentes de ácido gálico por interpolación de una curva de calibración $(1 \mathrm{mg} / \mathrm{mL}$ ) y se expresaron como $\mathrm{mg}$ ácido gálico/100 g de extracto. 


\section{8. Cuantificación de antocianos totales}

Para determinar el contenido de antocianos de los subproductos bajo estudio se siguió la metodología descrita por Giusti y Wrolstad (2001). La absorbancia final se obtuvo mediante la ecuación 1:

$$
\mathrm{A}=\left(\mathrm{A}_{510 \mathrm{~nm}}-\mathrm{A}_{700 \mathrm{~nm}}\right) \mathrm{pH} 1.0-\left(\mathrm{A}_{510 \mathrm{~nm}}-\mathrm{A}_{700 \mathrm{~nm}}\right) \mathrm{pH} 4.5
$$

El contenido de antocianos se expresó como miligramos equivalentes a cianidina-3-glucósido por 100 gramos de muestra.

\section{9. Cuantificación de flavonoides}

Para sacar la concentración de flavonoides totales se aplicó el método adaptado por Arvouet-Grand et al. (1994), el cual se basa en una reacción colorimétrica entre los flavonoides y el tricloruro de aluminio cuyo producto es un compuesto colorido que tiene un máximo de absorción a $420 \mathrm{~nm}$. Para cuantificar los flavonoides totales se preparó una curva de calibración con soluciones de concentración conocida de quercetina entre 5 y 50 ppm para obtener la ecuación de la recta. El resultado se expresó como mg equivalentes de quercetina en $100 \mathrm{~g}$ de residuo seco ( $\mathrm{mg}$ EQ/100 g de residuo seco).

\section{10. Contenido de $\beta$-carotenos}

Se pesaron $2 \mathrm{~g}$ de la muestra y luego se homogeneizó con $20 \mathrm{~mL}$ de una mezcla acetona-etanol (1:1). Se dejó reposar por 24 horas a $4{ }^{\circ} \mathrm{C}$. Después de ese tiempo, se filtraron las soluciones en un embudo con papel filtro y el sobrenadante se recogió en un vaso de precipitado con el debido cuidado de proteger la muestra de la luz. Se transfirió lo filtrado a balones volumétricos de $100 \mathrm{~mL}$ y se llevaron a volumen con solvente para carotenoides (acetona-etanol).

Se transfirió la solución a un embudo de separación, se adicionó $50 \mathrm{~mL}$ de hexano y $25 \mathrm{~mL}$ de agua y luego se agitó vigorosamente. Se dejó reposar por 30 minutos para que se dé la separación de fases. Se midieron las absorbancias de la fase orgánica usando una longitud de onda de $470 \mathrm{~nm}$. Para obtener el total de carotenos se realizó una curva de calibración con $\beta$-caroteno estándar de 100 ppm. La cuantificación se aplicó a la ecuación de la recta tomando en cuenta el peso en gramos de la muestra seca y el volumen total del extracto. Los resultados se expresaron como mg de $\beta$-carotenos/ $100 \mathrm{~g}$ de muestra.

\section{Determinación de la CaPaCidad antioxidante}

Método ABTS: fue desarrollado por Re et al. (1999) y descrito por Kuskoski et al. (2005). Para el método de ABTS se preparó una solución compuesta por ABTS $7 \mathrm{mM}$ y persulfato de potasio $0.45 \mathrm{mM}$. La mezcla se dejó reposar en la oscuridad durante 16 horas a temperatura ambiente. La solución stock se almacenó a temperatura ambiente en la oscuridad. Cada vez que era necesario se diluía la solución stock con etanol hasta alcanzar una absorbancia de $0.7 \pm 0.02$ a $734 \mathrm{~nm}$. Para el análisis de las muestras, se tomaron $3.9 \mathrm{~mL}$ de la solución de ABTS con absorbancia de $0.7 \pm 0.0$ y se mezcló con $0.1 \mathrm{~mL}$ de muestra. La absorbancia medida se realizó a $754 \mathrm{~nm}$ al minuto y a los siete minutos. La capacidad antioxidante se expresa como porcentaje de captación de radicales libres. 


\section{Análisis estadístico}

Se realizó un análisis estadístico multifactorial con tres repeticiones, se aplicó un ANOVA y la prueba de LSD para observar diferencias significativas entre las variables de respuestas por medio del programa Statgraphics Centurión XV versión 15.2.0.

\section{Resultados Y Discusión}

En el cuadro 1 se muestran los resultados del análisis bromatológico de las muestras estudiadas. En cuanto a la humedad, el polvo de cáscaras con mayor contenido fue el de la zanahoria con un 23\% y por debajo de ella se encuentra la papaya con 21.27\%; sin embargo, no existe diferencia significativa entre ellas $(p \leq 0.05)$. Las de menor cantidad son las de la cáscara de lima y la de berenjena (16.66 y $13.55 \%$ respectivamente). El contenido de humedad es un valor que influye en las características composicionales, pues depende del grosor de la cáscara, así como del tiempo y temperatura de secado al que fueron sometidos durante su procesamiento (Cruz, 2002).

\section{CUADRO 1}

Análisis bromatológicos de cáscaras de frutos

\begin{tabular}{|lrccccc}
\hline Análisis (\%) & \multicolumn{7}{c}{ Grasa } & Fibra & \multicolumn{1}{c}{ ELN } \\
\hline Muestras & Humedad & \multicolumn{1}{c}{ Ceniza } & Proteína & Grasa & \\
\hline Lima & $16.66 \pm 0.63^{\mathrm{a}}$ & $5.84 \pm 0.39 \mathrm{a}$ & $1.11 \pm 0.024^{\mathrm{a}}$ & $4.237 \pm 0.02^{\mathrm{a}}$ & $0.439 \pm 0.233^{\mathrm{a}}$ & $72.16 \pm 1.23^{\mathrm{a}}$ \\
Berenjena & $13.55 \pm 0.75^{\mathrm{a}}$ & $9.21 \pm 0.21^{\mathrm{b}}$ & $1.50 \pm 0.091^{\mathrm{b}}$ & $1.21 \pm 0.44^{\mathrm{a}}$ & $1.88 \pm 0.635^{\mathrm{b}}$ & $63.62 \pm 3.3^{\mathrm{b}}$ \\
Zanahoria & $23 \pm 0.20^{\mathrm{b}}$ & $12.97 \pm 0.29 \mathrm{c}$ & $0.67 \pm 0.028^{\mathrm{c}}$ & $1.88 \pm 0.169^{\mathrm{a}}$ & $0.409 \pm 0.099 \mathrm{a}$ & $61.063 \pm 0.21^{\mathrm{b}}$ \\
Papaya & $21.27 \pm 0.73^{\mathrm{b}}$ & $10.45 \pm 0.27 \mathrm{bc}$ & $2.23 \pm 0.19 \mathrm{~d}$ & $3.09 \pm 0.25^{\mathrm{a}}$ & $0.643 \pm 0.262^{\mathrm{a}}$ & $62.69 \pm 2.51^{\mathrm{b}}$ \\
\hline
\end{tabular}

Fuente: elaboración propia.

Nota: letras diferentes dentro de una misma columna representan diferencias significativas (LSD, $p<0.05$ ).

El contenido de cenizas en las cáscaras hace que puedan considerarse como fuentes potenciales de minerales. Al respecto, los resultados indican que la lima tiene un menor porcentaje y la de la zanahoria posee el de mayor contenido (12.97\%). Este porcentaje de cenizas en zanahoria resultó mayor al reportado por Chávez Zepeda et al. (2009) con 10.96\%. Habría que considerar que el contenido de cenizas también puede variar de acuerdo con el fruto, estado de madurez, variedad y temporada de cosecha, así como por las condiciones de cultivo (Priego, 2007).

La fracción de los compuestos nitrogenados solubles está formada por un 50\% de aminoácidos libres en promedio. Todos los demás compuestos nitrogenados son bastante escasos. También cabe resaltar que la mayor parte de la fracción proteica, la cual se encuentra sometida a grandes cambios dependientes de la clase de fruta y grado de madurez, está constituida por enzimas (Berlitz y Grosch, 1997). El porcentaje de proteína para la mayoría de los subproductos presentaron un valor entre 1 y $2 \%$, donde la cáscara con el mayor porcentaje es la papaya con $2.23 \%$ y el menor fue para la zanahoria con $0.670 \%$. Este contenido es mucho menor al encontrado por Chántaro et al. (2008), que se encuentra en $9.75 \%$.

En cuanto a la berenjena, el contenido de fibra encontrado en la cáscara resultó mayor respecto al porcentaje reportado en la pulpa: $1.50 \%$ y $1.2 \%$ en pulpa (Varela et al., 1995), lo cual es diferente respecto a la cáscaras de lima, zanahoria y papaya. La importancia del contenido de proteína en la cáscara radica sobre todo en la fracción que pueda cuantificarse como proteína indigerible debido a que si es resistente a la acción enzimática, podría formar parte de la fibra dietética (Priego, 2007). 
En cuanto al contenido de fibra, solamente la berenjena presentó un valor superior a 1\% y el resto no alcanzó ni el $1 \%$ en fibra. La diferencia que existe entre los datos obtenidos y los reportados por otros autores puede atribuirse a que se trata de diferentes variedades, además de que el clima, la temperatura, luminosidad y suelo son factores que también contribuyen a tal efecto (Serna y Torres, 2015).

La fracción lipídica de las frutas comprende acilgliceridos, glicolipidos, fosfolípidos, carotenoides, triterpenoides y ceras (Berlitz y Grosch, 1997). La cáscara de lima resultó la más alta en contenido de extracto etéreo con un 4.23\%, mientras que el resto de los subproductos fue inferior, ya que no sobrepasó 5\%; estadísticamente no hay diferencia significativa entre los valores encontrados en las cáscaras de los cuatro frutos. El contenido lipídico en cáscara de papaya se asemeja al reportado por Cervantes et al. (2016) con 1.8\%.

El extracto libre de nitrógeno obtenido fue significativamente mayor en la harina de lima (72.61\%), le sigue la de berenjena que presenta un promedio de 63.62\%; sin embargo, no existe diferencia significativa entre la berenjena, zanahoria y papaya. El valor del ELN es una medida indirecta de los carbohidratos solubles o digeribles (Vasco, 2008). Se puede decir que los carbohidratos de las frutas son azúcares y en frutas cítricas están conformados por monosacáridos (glucosa y fructosa), oligosacáridos (sacarosa) y polisacáridos (celulosa, almidón, hemicelulosa y pectinas) (Repo de Carrasco y Encina Zelada, 2008). Debido al alto contenido de carbohidratos de la cáscara que se obtuvo en esta investigación es posible tener en cuenta estos componentes como un posible estudio en las cáscaras.

Los contenidos en carbohidratos y proteína en las cáscaras de berenjena y papaya indican que este residuo de la agroindustria podría ser utilizado como fuente de carbono y nitrógeno en sustratos de fermentación de origen orgánico. Como se sabe, en la industria de las fermentaciones se utilizan fuentes inorgánicas de nitrógeno, las cuales tienden a ser costosas en los medios de cultivo y las convierte en un limitante para la elaboración de productos orgánicos obtenidos por fermentación (Serna y Torres, 2015).

\section{Cuantificación de sustancias bioactivas}

Las muestras de lima, zanahoria y papaya presentaron una diferencia significativa en el contenido de compuestos fenólicos respecto a la berenjena, que fue la que presentó los mayores valores como se puede apreciar en el cuadro 2. Las frutas, aparte de ser fuentes importantes de ciertas vitaminas, contienen otros componentes, principalmente pigmentados, como son los compuestos fenólicos que poseen capacidad antioxidante y también son capaces de inhibir los radicales libres. Los estudios epidemiológicos llevados a cabo en la última década sugieren que el consumo de una dieta rica en estos compuestos fenólicos está asociado a la disminución del riesgo de sufrir enfermedades cardiovasculares (Heiss et al., 2006), ciertos tipos de cáncer (Weng et al., 2012) y enfermedades degenerativas (Vauzour et al., 2010).

CUADRO 2

Contenido de compuestos bioactivos presentes en cáscaras de diferentes frutos

\begin{tabular}{|lccccc}
\hline \multicolumn{5}{|l}{ Análisis (mg/ 100g) } \\
\hline Muestras & Polifenoles totales & Antocianinas & $\beta$-carotenos & Flavonoides & $\begin{array}{c}\text { Capacidad } \\
\text { antioxidante }(\%)\end{array}$ \\
\hline Lima & $4500.86 \pm 145.47^{\mathrm{a}}$ & $15.023 \pm 1.42^{\mathrm{a}}$ & $8.01 \pm .082^{\mathrm{a}}$ & $252.53 \pm 18.11^{\mathrm{a}}$ & $99.85 \pm 0.18^{\mathrm{a}}$ \\
Berenjena & $10289.72 \pm 187.14 \mathrm{~b}$ & $59.635 \pm 2.02^{\mathrm{b}}$ & $5.33 \pm 0.29 \mathrm{~b}$ & $292.58 \pm 3.52^{\mathrm{a}}$ & $97.90 \pm 0.21^{\mathrm{b}}$ \\
Zanahoria & $4960.18 \pm 341.44^{\mathrm{a}}$ & $34.81 \pm 1.06^{\mathrm{a}}$ & $28.3 \pm 0.62^{\mathrm{c}}$ & $90.48 \pm 9.75^{\mathrm{b}}$ & $99.70 \pm 0.21^{\mathrm{a}}$ \\
Papaya & $4798.93 \pm 242.50^{\mathrm{a}}$ & $11.01 \pm 067^{\mathrm{a}}$ & $42.13 \pm 0.671^{\mathrm{d}}$ & $385.27 \pm 19.62^{\mathrm{c}}$ & $99.63 \pm 0.106^{\mathrm{a}}$ \\
\hline
\end{tabular}

Fuente: elaboración propia.

Nota: letras diferentes dentro de una misma columna representan diferencias significativas (LSD, $p<0.05$ ). 
Aunque el contenido de sustancias fenólicas es un indicativo de la actividad antioxidante de una muestra, es conveniente analizar el contenido de algunos tipos de fenoles con actividad antioxidante elevada como es el caso de los flavonoides y en específico de las antocianinas. Además de nutrientes esenciales, como vitaminas y minerales, las frutas pueden presentar pigmentos antociánicos de demostrada actividad antioxidante. El contenido de antocianinas fue significativamente más alto en la cáscara de berenjena, seguido de la zanahoria y papaya; la lima fue la que presentó menor contenido de estos pigmentos. De igual modo, se puede observar que existe una correlación positiva entre el contenido de polifenoles y el de antocianinas en las cáscaras evaluadas. En cuanto al contenido de antocianinas en cáscara de berenjena, Heras et al. (2013) encontraron $62 \mathrm{mg}$ de antocianinas/100 $\mathrm{g}$ de cáscara, resultado ligeramente mayor al encontrado en este trabajo.

Los carotenoides constituyen un grupo importante en la dieta humana, pues, además de su actividad vitamínica, cumplen otras actividades biológicas que incluyen la capacidad antioxidante, el filtrado de la luz ultravioleta, la modulación de la función inmune y la regulación de la diferenciación y proliferación celular (Ordoñez y Ledezma, 2013). Aunado a la propiedad antioxidante, los carotenoides pueden ser utilizados como pigmentos naturales, ya que son los responsables de los colores naranja, amarillo y rojo en los alimentos; un ejemplo es la utilización del achiote y de la paprika en la industria alimentaria (Zeni et al., 2011).

El contenido de carotenoides en la cáscara de papaya resultó significativamente mayor, le siguió el de las cáscaras de zanahoria, lima y berenjena. Estudios similares han demostrado mayor contenido de carotenoides en residuos de papaya al compararlos con los de piña, naranja y guayaba (Ordóñez et al., 2014). Sin embargo, los resultados presentados por Ordoñez et al. (2014) y por González (2013) son inferiores a los encontrados en este trabajo ( 5.88 y $1.35 \mathrm{mg} / 100 \mathrm{~g}$ respectivamente).

Las diferencias entre los resultados obtenidos en este trabajo y los de otros autores respecto a la concentración de carotenoides puede ser consecuencia de la variación genética y de factores de manejo de pre y poscosecha de los frutos, tal como ha sido discutido por Dumas et al. (2003).

La relación entre el color y los carotenoides en las muestras analizadas se debe principalmente a la presencia de sistemas de dobles enlaces conjugados de la cadena polienoica de los carotenoides que permite formar un cromóforo (parte de la estructura responsable de la absorción de luz visible y por tanto del color del compuesto), cuya capacidad de absorción de luz da lugar a los llamativos y característicos colores de estos pigmentos (anaranjado-amarillo), y el número de dobles enlaces conjugados y la presencia de diferentes grupos funcionales determinarán en última instancia las características espectroscópicas propias de cada pigmento (Moreno et al., 2012). Además, cuando el fruto comienza a tornarse amarillo ocurre un mayor aumento en azúcares por lo que se acelera la síntesis de estos compuestos hasta alcanzar la coloración muy amarilla (Torres et al., 2013), la cual se nota más en la cáscara de papaya que en la de zanahoria.

El contenido de flavonoides en las diferentes cáscaras resultó mayor en la de papaya, seguida de la berenjena y de la lima; la de zanahoria fue significativamente menor en comparación con el resto. Moo (2012) reportó una concentración promedio de flavonoides en cáscara de lima de $222.3 \mathrm{mg}$ EQ/100 g de residuo seco, y logró valores tan altos como $282.5 \mathrm{~g} / 100 \mathrm{~g}$ dependiendo de la localidad en la cual se adquirió el fruto. Las cáscaras o pieles de las frutas contienen una variedad de ácidos hidroxicinámicos (HCA), flavan-3-oles (monoméricos y oligoméricos), flavonoles y sus conjugados, dihidroxichalconas y procianidinas (Rodríguez, 2006), entre los cuales está la cáscara de tomate de árbol, la carambola y una gran variedad de cítricos (Muñoz et al., 2009).

Los flavonoides son los compuestos polifenólicos mejor estudiados que se caracterizan por tener una estructura de tres anillos formada por dos centros aromáticos y un heterociclo central oxigenado. Dentro de los flavonoides se incluyen a las flavonas, flavanonas, catequinas y antocianinas, y se ha demostrado que ellos previenen la agregación plaquetaria e inducen la relajación muscular; junto con los proteoglicanos, los flavonoides ejercen un efecto inhibitorio de los síntomas alérgicos. Algunos flavonoides como la procianidina B1 y el resveratrol presentes en extractos de semillas de uva y en la uva como tal pueden aumentar la capacidad cerebral y la longevidad (Drago Serrano et al., 2006). 
La actividad antioxidante es un parámetro que determina qué tanto el compuesto antioxidante evita que su sustrato se oxide. Por otro lado, el ABTS•+ es soluble en solventes acuosos y orgánicos, lo cual lo hace un método apto para determinar la capacidad antioxidante hidrofílica y lipofílica de extractos y fluidos biológicos (Schlesier et al., 2002; Prior et al., 2005); por ello, se le ha llamado en ocasiones capacidad antioxidante total. El cuadro 2 muestra los valores para las diferentes cáscaras empleando el radical ABTS $\bullet+$. Los valores obtenidos indican hasta un 99.85\% de inhibición para este radical en las cáscaras de lima, 99.70\% para la zanahoria, 99.62\% en papaya y $97.90 \%$ para la berenjena. Esta elevada capacidad antioxidante es primordial para la salud porque puede prevenir enfermedades como el cáncer y otras enfermedades degenerativas, así como algunas actividades biológicas (antiinflamatoria o antidiabética, por mencionar algunas).

La capacidad antioxidante en las cáscaras de lima, papaya y zanahoria fue mayor y no presentó diferencia significativa entre cada una, pero sí respecto a la de berenjena.

El análisis de regresión lineal demostró una correlación estadísticamente significativa entre la actividad antioxidante y el contenido de polifenoles $(r=-0.9911, p<0.05)$, así como entre la actividad antioxidante y las antocianinas $(r=-0.8757, p<0.05)$, una correlación moderadamente fuerte entre el ABTS y los $\beta$-carotenos $(r$ $=0.5063, p<0.05)$ y una relación débil entre las variables ABTS y flavonoides $(r=0.2172, p<0.05)$. Aunque las cáscaras de berenjena tienen altos contenido de polifenoles, su baja contribución en la actividad antioxidante medida por ABTS sugiere que deben existir otros compuestos que impacten de manera más significativa, como las antocianinas.

\section{Conclusiones}

Referente a la parte bromatológica, se encontró que el mayor contenido de proteína lo presentó la cáscara de papaya, en tanto que la fibra fue mayor en la de berenjena y la de lima obtuvo la mayor cantidad de extracto etéreo y ELN. Todas las cáscaras presentaron actividad antioxidante elevada, medida como porcentaje de inhibición de radicales, que fue mayor en la lima, papaya y zanahoria, en tanto que el mayor contenido de polifenoles y antocianinas fue el encontrado en la de berenjena, y en la de papaya se obtuvieron valores significativos de $\beta$-carotenos y flavonoides.

Los resultados obtenidos indican que con las cáscaras se pueden aplicar procesos sencillos que permitan la extracción de moléculas de alto valor, lo cual puede traducirse en oportunidades de negocio. La utilización de cáscaras de frutas constituye una alternativa para el manejo sustentable de desechos provenientes de la agroindustria, de los cuales destaca el valor agregado que se les puede dar como fuente de compuestos bioactivos de interés por presentar un mayor potencial para reutilizarse en la alimentación humana o en la industria farmacéutica.

\section{SugerenCIAS Y PROSPECTIVA}

La cantidad desechos orgánicos, proveniente principalmente de frutas y hortaliza, incrementa día con día. Este hecho representa un problema en cuanto al costo de recolección o disposición, así como de contaminación ambiental debido a que se tiran a cielo abierto; sin embargo, estos desechos de bajo costo, abundantes y disponibles pueden ser una fuente importante de compuestos debido a sus propiedades nutrimentales (fibra, minerales y proteínas) y a la presencia de compuestos bioactivos que permitirían usarlos como antioxidantes y prebióticos. Esto implica una necesidad por estudiar estos residuos y así conocer a detalle su composición nutrimental y antioxidante. Con ello, obtener moléculas de interés comercial a bajo precio que al ser incorporadas al alimento le confieran propiedades funcionales y le den valor agregado. No obstante, su composición depende de diferentes factores como la variedad, el tipo de suelo, la cosecha y el procesamiento industrial, entre otros. Por lo tanto, los usos para dichos residuos requiere del conocimiento de su composición química, las propiedades de sus componentes y los grupos funcionales que los constituyen, así como el desarrollo de tecnologías apropiadas para lograr 
una mayor efectividad en su aprovechamiento. Se sugiere ahondar en el estudio de estos subproductos, cáscaras y semillas y más en aquellos que sean nativos de la región y de los cuales se tiene muy poca o nula la información. $\mathrm{Al}$ respecto, en este artículo se analizaron las cáscaras de papaya, lima, zanahoria y berenjena, productos comunes en el estado de Yucatán, que se encuentran disponibles la mayor parte del año. De igual manera, es necesario analizar a detalle cada uno de los componentes que le confieren esa actividad antioxidante, así como seguir en la búsqueda de posibles aplicaciones de estos materiales, lo cual pudiera abarcar la industria de los alimentos al ser usados como enriquecedores, pigmentos o como fuente para el desarrollo de recubrimientos comestibles, así como su posible utilización en la industria farmacéutica.

\section{Agradecimientos}

Los autores agradecen al Tecnológico Nacional de México por el apoyo financiero recibido para el desarrollo de la investigación a través del proyecto con registro 5770.16-P.

\section{REFERENCIAS}

Angulo, P., Díaz, D., Espinoza, J., Fernández, V., Figueroa, M. y Galarza, A. (2001). Implicaciones farmacológicas y toxicológicas del óxido nítrico en la inflamación intestinal II: enteritis inducida por AINES como modelo experimental para el científico de la flora medicinal peruana. Revista de Ciencias Veterinarias, $17(3), 21-26$

Arvouet-Grand, A., Vennat, B., Pourrat, A., \& Logret, P. (1994). Standarisation the extrain de propolis et identification de principaux constituants. Journal the Pharmacie the Belgique, 49, 462-468.

Berlitz, H. D., y Grosch, W. (1997). Quimica de los alimentos (segunda edición). Zaragoza: Acribia.

Cayuela, M., Millner, P., Meyer, S., \& Roig, A. (2008). Potential of olive mill waste and compost as biobased pesticides against weeds, fungi, and nematodes. Science of the Total Environment, 399, 11-18

Cervantes Delfín, K., Cruz López, A. y Campos Mondragón, M. (2016). Subproductos obtenidos a partir de distintas cáscaras de fruta. Revista Iberoamericana de Producción Académica y Gestión Educativa, 4.

Chantaro, P., Devahastin, S., \& Chiewchan, N. (2008). Production of antioxidant high dietary fiber powder from carrot peels. Food Science and Technology, 41, 1987-1994.

Chávez Zepeda, L., Cruz Méndez, G., Gracia de la Caza, L., Díaz- Vela, J. y Pérez Chabela, M. (2009). Utilización de subproductos agroindustriales como fuente de fibra para productos cárnicos. Nacameh, 3(2),71-82.

Cruz, S. (2002). Caracterización fisicoquimica, fisiológica y funcional de residuos fibrosos de cáscara de maracuyá (Pasiflora edulis) (tesis de maestría). Mérida: Universidad Autónoma de Yucatán.

Di Gioia, L., Sciubba, L., Setti, F., Luziatelli, M., Ruzzi, D., Zanichelli,G., \& Fava, F.(2007). Production of biovanillin from wheat bran. Enzyme and Microbial Technology, 41, 498-505.

Drago Serrano, M. D., López, L., \& Sainz, E. (2006). Componentes bioactivos de alimentos funcionales de origen vegetal. Revista Mexicana de Ciencias Farmacéuticas, 37, 58-68.

Dumas, Y., Dadomo, M., Di Lucca, G., \& Grolier, P. (2003). Review effects of environmental factors and agricultural techniques on antioxidant content of tomatoes. Journal of the Science of Food and Agriculture, 83, 369-382.

Giusti, M., \& Wrolstad, R. (2001). Current protocols in food analytical chemistry. New York: Wrolstad REE Jonh Wiley \& Sons. 
González, O. (2013). Aprovechamiento de residuos agroindustriales para la producción de alimentos funcionales: una aproximación desde la nutrición animal (tesis). Antioquía.

Heiss, C., Schroeter, H., Balzer, J., Kleinbongard, P., Matern, S., \& Sies, H. (2006). Endothelial function, nitric oxide, and cocoa flavanols. Journal of Cardiovascular Pharmacology, 47 (2), 28-35.

Heras, I., Alvis, A. y Arrazola, G. (2013). Optimización del proceso de extracción de antocianinas y evaluación de la capacidad antioxidante de berenjena (Solana melonera L.). Información tecnológica, 24(5), 93-102.

Kuskoski, M., Asuero, A. y Troncoso, A. (2005). Aplicación de diversos métodos químicos para determinar actividad antioxidante en pulpa de frutos. Ciência e Tecnologia de Alimentos, 25(4), 726-732.

Méndez, R. (1995). Aprovechamiento de subproductos agropecuarios. Santafé de Bogotá: Unisur.

Moo, M., Vargas, L., Cuevas, L., Moo. V., Sauri, E. (2012). Evaluación del contenido de flavonoides totales en residuos cítricos. Memorias en extenso del VII Congreso Internacional de Ingeniería Bioquímica y $\mathrm{X}$ Jornadas Científicas de Biomedicina y Biotecnología Molecular. Ixtapa-Zihuatanejo, México.

Moreno, J., Gorriti, M., Flores, M. y Albarracín, V. (2012). Microbiología ambiental y ecología microbiana en el estudio de microorganismos en ambientes extremos. REDUCA (Biología), 5(5), 94-109.

Mullen, W., Marck, S., \& Crozier, A. (2007). Evaluation of phenolic compounds in commercial fruit juices and fruit drinks. Journal Agriculture Food Chemistry, 55(8), 3148-3157.

Muñoz, A. J., Ramos Escudero, F., Alvarado Ortiz, C. U., Castañeda, B. C., y Lizaraso, F. C. (2009). Evaluación de compuestos con actividad biológica en cáscaras de Camu Camu, Guinda, Tomate de árbol y Carambola cultivadas en Perú. Revista de la Sociedad Química del Perú, 75(4), 431-438.

Oliveira, A., Barros, C., Silva, E., Henriques M., Paes de Barros, M., Marinho, M., \& Fonseca, O. (2009). Total Phenolic content and free radical scavenging activities of methanolic extract powders of tropical frutis residues. Food Chemestry, 115, 469-475.

Ordóñez, L., Hurtado, P., Ríos, O., \& Arias, M. (2014). Total concentration of carotenoids in tropical fruits waste. Producción + Limpia, 9(1), 91-98.

Ordoñez, L., \& Ledezma, D. (2013). Lycopene concentration and physico-chemical properties of tropical fruits. Food and Nutrition Sciences, 4, 758-762.

Parawira, W., Read, J. S., Mattiasson, B., \& Björnsson. (2008). Energy production from agricultural residues: High methane yields in pilot stage anaerobic digestión. Biomass and Bioenergy, 32, 44-50.

Priego, N. (2007). Obtención de fibra dietética a partir de sáculos de naranja aplicando un tratamiento con vapor (tesis). México: Universidad Tecnológica de la Mixteca.

Prior, R. L., Wu, X., \& Schaich, K. (2005). Standardized methods for the determination of antioxidant capacity and phenolics in foods and dietary supplements. Journal Agriculture Food Chemistry, 53, 4290-4302.

Re, R., Pellegrini, N., Proteggente, A., Pannala, A., Yang, M., \& Rice Evans, C. (1999). Antioxidant activity applying an improved ABTS radical cation decolorization assay. Free Radical Biology and Medicine, 26, 1231-1237.

Repo de Carrasco, R. y Encina Zelada, C. (2008). Determinación de la capacidad antioxidante y compuestos bioactivos de frutas nativas peruanas. Revista de la Sociedad Quimica del Perú, 74(2), 108-124.

Rivas Torres, B., Aliakbarian, B., Torre, P., Perego, P., Domínguez, J., Zilli, M., \& Converti, A. (2009). Vanillin bioproduction from alkaline hydrolyzate of corn cob by Escherichia coli JM109/pBB1. Enzyme and Microbial Technology, 44(3), 154-158.

Rodríguez, M. R., Picinelli, L. A., \& Suárez, V. B. (2006). Phenolic profile of Asturias (Spain) natural cider. zSchlesier, K., Harwat, M., Bohm, V., \& Bitsch, R. (2002). Assessment of antioxidant activity by using 
different in vitro methods. Free Radical Research, 36(2), 177-187.

Serna, L. y Torres, C. (2015). Potencial agroindustrial de cáscaras de mango de las variedades Keitt y Tommy Atkins (Mangifera indica). Acta Agronómica, 66(2), 110-115.

Torres, R., Montes, E., Pérez, O. y Andrade, R. (2013). Relación del color y del estado de madurez con las propiedades fisicoquímicas de frutas tropicales. Información Tecnológica, 24(3), 51-56.

Vasco, V. (2008). Determinación de parámetros fisicoquímicos de zanahoria amarilla (Daucus carota) (tesis de grado). Ecuador: Escuela Superior Politécnica de Chimborazo.

Varela, G., Moreiras, A. y Carbajal, M. (1995). Estudio nacional de nutrición y alimentación (ENNA). Instituto Nacional de Estadística.

Vauzour, D., Rodriguez, A., Corona, G., Oruna, M. J., \& Spencer, J. (2010). Polyphenols and human health: Prevention of disease and mechanisms of action. Nutrients, 2(11), 1106-1131

Weng, C. J., \& Yen, G. C. (2012). Chemopreventive effects of dietary phytochemicals against cancer invasion and metastasis: Phenolic acids, monophenol, polyphenol, and their derivatives. Cancer Treatment Reviews, 38(1), 76-87.

Wichi, H. P. (1988). Tumor development by butylated hydroxyanisole (BHA) from the prospective of effect on forestomach and oesophageal squamous epithelium. Food and Chemical Toxicology, 26(8), 717-723.

Zeni, J., Colet, R., Cence, K., Tiggemann, L., Toniazzo, G., Cansian, L., \& Valduga, E. (2011). Screening of microorganisms for production of carotenoids. CyTA-Journal of Food, 9(2), 160-166.

\section{BY-NC-ND}

